\title{
BMJ Open Acupuncture for patients with chronic pruritus: protocol of a systematic review and meta-analysis
}

\author{
Leixiao Zhang (1) , ${ }^{1}$ Yanli Deng, ${ }^{2}$ Junpeng Yao, ${ }^{1}$ Xianjun Xiao, ${ }^{1,3}$ Siyi Yu, ${ }^{1}$ \\ Yunzhou Shi, ${ }^{1}$ Hui Zheng (D) , ${ }^{1}$ Qianhua Zheng, ${ }^{1}$ SiYuan Zhou, ${ }^{1}$ Wei Cao, ${ }^{1}$ Ying Liu, ${ }^{1}$ \\ Pingsheng Hao, ${ }^{4}$ Ying $\mathrm{Li}^{1}$
}

To cite: Zhang L, Deng Y, Yao J, et al. Acupuncture for patients with chronic pruritus: protocol of a systematic review and meta-analysis. BMJ Open 2020;10:e034784. doi:10.1136/ bmjopen-2019-034784

- Prepublication history for this paper is available online. To view these files, please visit the journal online (http://dx.doi org/10.1136/bmjopen-2019034784).

LZ and YD contributed equally.

Received 08 0ctober 2019

Revised 28 April 2020

Accepted 24 June 2020

Check for updates

(c) Author(s) (or their employer(s)) 2020. Re-use permitted under CC BY-NC. No commercial re-use. See rights and permissions. Published by BMJ.

${ }^{1}$ Acupuncture and Tuina School, Chengdu University of Traditional Chinese Medicine, Chengdu, China

${ }^{2}$ Sichuan Second Chinese Medicine Hospital, Chengdu, China

${ }^{3}$ The People's Hospital of Jianyang City, Jianyang, China ${ }^{4}$ Affiliated Hospital of Chengdu University of Traditional Chinese Medicine, Chengdu, China

\section{Correspondence to}

Professor Ying Li; liying@cdutcm.edu.cn and Professor Pingsheng Hao; hpswl@126.com

\section{ABSTRACT}

Introduction Chronic pruritus (CP) frequently occurs in many skin and systemic diseases, and adversely affects quality of life. This systematic review aims to evaluate treatment effects of acupuncture on CP.

Methods and analysis An electronic and manual search will be conducted for all acupuncture treatments for $\mathrm{CP}$, from the inception date of predefined database up to 28 February 2020. Databases include PubMed, Embase, Springer, Web of Science, the Cochrane Library, the World Health Organization International Clinical Trial Registration Platform, the Chinese Medicine Database, the China National Knowledge Infrastructure, the Chinese Biomedical Literature Database, the China Science Journal Database and the Wanfang Database. Other sources, including existing systematic reviews, conference proceedings and reference lists of identified publications will also be searched. Additionally, any clinical randomised controlled trials related to acupuncture treatment for $\mathrm{CP}$, regardless of the publication status and language limitations, will be included. Study selection, data extraction and research quality assessments will be conducted independently by two researchers. The primary outcome measures include the Visual Analogue Scale, Urdu 5D-Itch Scale or other validated scales implemented after at least 2 weeks of treatment. Secondary outcomes include the effective rate, Quality of Life Scale (eg, the EQ-5D third level, the Dermatology Life Quality Index, etc.), Pittsburgh Sleep Quality Index, recurrence rate during the follow-up period and adverse events. If possible, meta-analyses will be performed using RevMan V.5.3 statistical software; otherwise, a descriptive analysis or subgroup analysis will be conducted. The results will be presented as the risk ratio of the binary data and the mean difference (MD) or standardised MD of the continuous data.

Ethics and dissemination This systematic review protocol does not require formal ethical approval because the data are not personalised. It will be published in peerreviewed journals and presented at international academic conferences.

PROSPERO registration number CRD42019136727.

\section{INTRODUCTION}

Description of the condition

According to the International Forum for the Study of Itch, chronic pruritus (CP) is
Strengths and limitations of this study

The study design adheres to all relevant guidelines for systematic reviews and meta-analyses.

- Only randomised controlled trials will be included.

- Language and publication dates will be unlimited.

- A sensitivity analysis will be performed.

- Different effectiveness evaluation criteria and acupuncture treatment methods may lead to heterogeneity.

defined as pruritus lasting 6 weeks or longer. ${ }^{1}$ It is a frequent symptom in many skin and systemic diseases in the general population, and the most current patients have a 3-8-year history of CP. ${ }^{2} \mathrm{CP}$ is associated with a severe pruritus sensation, which poses a significant burden on quality of life similar to that of chronic pain. ${ }^{2-4}$ Worldwide, the prevalence of acute pruritus ( $<6$ weeks) is $8.4 \% \cdot{ }^{45}$ Based on the European survey data, the prevalence of CP ( $>6$ weeks) is approximately $8.2 \%-22 \%$ in adults. ${ }^{26}$ However, in the elderly ( $>65$ years of age), the figure is likely to be $50 \%$ or higher. ${ }^{7}$ In the USA, Turkey and Thailand, the incidence of CP in the elderly is 29\%, $11.5 \%$ and $41 \%$, respectively. ${ }^{8-10}$ Patients evaluated for $\mathrm{CP}$ are more likely to be African or Asian than of other ethnicities (20\% vs $14 \%) .{ }^{11}$ Interestingly, about $12.8 \%$ of patients suffer from CP during the winter months. ${ }^{9}$ Female patients seem to have more pronounced symptoms. ${ }^{912} 13$

It is estimated that about $18 \%$ of women will develop CP during pregnancy. ${ }^{14}$ Notably, CP is the leading symptom of specific pregnancy dermatoses such as polymorphic eruption of pregnancy, pemphigoid gestationis, intrahepatic cholestasis of pregnancy and atopic eruption of pregnancy. ${ }^{14}{ }^{15}$ The prevalence of $\mathrm{CP}$ in adolescents is $8.8 \%$. Moreover, CP is as strongly associated with suicidal ideation among adolescents as is chronic pain. ${ }^{16}$ It is 
also associated with mental distress, the female sex and sociodemographic factors. ${ }^{17}$

The aetiology of CP is classified as follows: I, dermatological; II, systemic; III, neurological; IV, somatoform; $\mathrm{V}$, mixed origin; and VI, others. ${ }^{1}$ However, the origin of $\mathrm{CP}$ is unclear in $8 \%-15 \%$ of patients with this diagnosis. ${ }^{4}$ Skin or systemic diseases may cause CP. For example, all patients with urticaria and $80 \%$ of patients with psoriasis have CP. ${ }^{18-20}$ Systemic diseases such as primary biliary cirrhosis have an $80 \%-100 \%$ association. Additionally, more than $30 \%$ of patients with Hodgkin's lymphoma have CP symptoms. There are also systemic diseases that can cause CP including, but not limited to, chronic renal insufficiency, hyperparathyroidism, iron deficiency, autoimmune deficiency disorder, myelodysplastic syndrome, multiple sclerosis, brain tumours, depression and hallucinosis. ${ }^{3}$

Almost any drug may induce CP by various pathomechanisms. Drugs that may induce or maintain CP include angiotensin-converting enzyme inhibitors, antiarrhythmic agents, antidepressants, antidiabetic drugs, beta blockers, immunosuppressive drugs and neuroleptics. ${ }^{3}$ Drug-induced hepatic oxygenation or cholestasis and drugs that cause dryness or phototoxicity may produce CP on normal skin. ${ }^{21}$ For example, hydroxyethyl starch, a compound used for fluid restoration, can induce chronic generalised or localised pruritus. ${ }^{22}$

According to the European guidelines, CP treatments include the use of simple and effective wet and cold wraps, the application of lotio alba, avoiding contact with allergens, stopping medications that may cause CP and possible surgical treatment. ${ }^{3}$ In addition, systemic H1 antihistamines, corticosteroids, capsaicin, gabapentin, tranquillisers, antidepressants and other drugs can also be used throughout treatment. ${ }^{32324}$ However, long-term drug use can cause many side effects. For example, corticosteroids can lead to dry and atrophic skin. H1 antihistamines can cause headaches, insomnia, lethargy, fatigue, dry mouth and allergies. ${ }^{25}{ }^{26}$ The lack of stable and effective treatment increases healthcare costs. The American Academy of Dermatology's recent national Burden of Skin Disease report revealed a pruritus-specific total medical cost of US\$294 million. ${ }^{27}$

Notably, there are an increasing number of recent publications on the use of acupuncture to treat CP, such as uremic pruritus, chronic idiopathic pruritus and pruritus induced by intrathecal morphine. ${ }^{28-30}$ The positive effects of acupuncture on CP are mentioned in the European S2k guidelines on $\mathrm{CP}^{31}$ While recent systematic reviews have been published in 2015 and 2018 on acute pruritus ${ }^{32}$ and uremic pruritus, respectively, ${ }^{33}$ neither review was limited to acupuncture and CP. Therefore, this systematic review aims to evaluate the effectiveness and safety of acupuncture therapy for $\mathrm{CP}$, based on the analysis of articles published from the inception date of specific, predefined databases up to 28 February 2020 regardless of the publication status and language.

\section{Description of the intervention}

For more than 5000 years, traditional Chinese medicine (TCM) has become an inseparable part of traditional Chinese culture. Its unique theoretical system was gradually formed 3000 years ago. Acupuncture, an important part of TCM, involves the insertion of fine needles into specific anatomical locations (acupuncture points) believed to be involved with specific disorders. For over 2500 years, acupuncture has been used to treat CP with few adverse reactions. ${ }^{32} 34$

\section{How the intervention works}

According to the TCM theory, Qi and blood are the most basic substances that constitute the human body and maintain life. If the balance of Yin and Yang is disturbed, a Qi deficiency, which reflects an excessive or insufficient amount of blood, can lead to pain, pruritus, insomnia and other diseases. ${ }^{35}$ Acupuncture is a procedure in which specific body areas, known as the acupoints (also called meridian points), are pierced with fine needles for therapeutic purposes. ${ }^{35}$ Acupuncture treats illness by recreating the balance between the Yin and Yang forces and restoring normal Qi, blood and bodily fluids. This is achieved through the stimulation of different acupoints, which govern different parts of the body and their interactions. ${ }^{36}$ Although systemic therapies such as acupuncture have shown promise for relieving CP, its mechanisms remain unclear. According to reports, acupuncture can alleviate pruritus and reduce pruritusevoked activation in the insula, putamen, premotor and prefrontal cortical areas. ${ }^{37}$ Acupuncture promotes the selective release of opioids in the spinal cord, which block the impulses associated with pruritus transmitted from the periphery by slow conductive C fibres. ${ }^{38}$ Additionally, an activated parasympathetic nervous system, as well as the functional connectivity of the putamen and the posterior part of the midcingulate cortex, could be considered as factors related to the antipruritic effect of acupuncture. $^{39}$

\section{Why would this review be important?}

To improve CP symptoms, a systematic review is needed to provide evidence for clinical treatment. However, the effectiveness of acupuncture needs to be confirmed with additional clinical studies conducted among different ethnic samples. In the past 4 years, at least five random clinical trials (RCTs) have been published and their inclusion in this review will hopefully provide a clear picture of the role of acupuncture in CP treatment. ${ }^{3040}$ We hope that this systematic review will provide a more substantial conclusion.

\section{OBJECTIVES}

To further systematically assess the effectiveness and safety of acupuncture in $\mathrm{CP}$ treatment. 


\section{METHODS AND ANALYSIS}

\section{Review design}

This protocol report is structured according to the Preferred Reporting Items for Systematic Reviews and Meta-Analyses (PRISMA) Protocols statement guidelines. ${ }^{41}$ The review will be implemented in accordance with the PRISMA statement guidelines. ${ }^{42}$

\section{Inclusion criteria for study selection}

Types of studies

All articles published from the inception date of each database up to 28 February 2020 will be included, without language and publication type limitations. However, animal mechanism studies, case reports, self-controlled studies, non-RCTs, randomised crossover studies and quasirandomised trials will be excluded.

\section{Types of participants}

The review will include trials that recruited participants who met diagnostic criteria or had CP symptoms associated with specific diseases ( $>6$ weeks including, but not limited to, CP in inflamed and non-inflamed skin, CP with kidney disease, $\mathrm{CP}$ with hepatic diseases and $\mathrm{CP}$ with metabolic and endocrine diseases). All eligible participants, regardless of age, race, sex, ethnicity, education and economic status, will be included in this review. Pregnant women, nursing mothers and people with other serious illnesses will be excluded. ${ }^{3}$ For example, laryngeal oedema caused by allergies can be life-threatening and should be treated as an emergency. ${ }^{43}$ If the patient does not inform the doctor of a prior diagnosis of liver and spleen enlargement, emphysema or other diseases, acupuncture may result in adverse reactions. For patients with unstable blood pressure and grade 3 hypertension, acupuncture may cause a transient increase in blood pressure. ${ }^{44}$ Acupuncture is also not advised in the presence of a skin infection, ulcer, scar, tumour, coagulopathy or serious mental illness. ${ }^{40}$

\section{Types of interventions}

Acupuncture refers to a method of stimulating acupuncture points, including manual acupuncture, dermal needles, plum blossom needles, ear acupuncture, electroacupuncture or fire needles. Other methods such as acupressure, moxibustion, laser acupuncture, pharmacoacupuncture, dry needling or transcutaneous electrical nerve stimulation will be excluded. Sham acupuncture can serve as a control, and may be: (1) performed at selected acupoints (the needles are inserted superficially without manipulation at the same selected acupoints in the experimental group); (2) performed at nonacupoints (the method is performed $1-3 \mathrm{~cm}$ away from the selected acupoints in the same neural segment but at non-acupoints, or at non-acupoints in distant areas); (3) performed at inappropriate acupoints (needling at acupoints that are inactive, serving as a way to test acupoint specificity) or (4) non-penetrating (tapping a blunt needle or guide tube to the dermal surface without skin penetration on the selected acupoints or nonacupoints). A pseudointervention, such as a mock transcutaneous nerve stimulation or the use of an inactivated laser apparatus, may also act as a control. ${ }^{45}$

The following comparisons will be evaluated: (1) acupuncture versus no treatment; (2) acupuncture versus placebo or sham acupuncture; (3) acupuncture versus other active therapies and (4) acupuncture in addition to active therapy versus the same active therapy. RCTs in which acupuncture is only compared with another form of acupuncture, or a different type of TCM (such as Chinese herbal medicine), will be excluded. Acupuncture treatment will be evaluated based on the training and education of the acupuncturist, clinical experience, acupuncture frequency, treatment duration and treatment frequency. ${ }^{46}$

\section{Types of outcome measures}

\section{Primary outcomes}

The primary outcomes include a Visual Analogue Scale, ${ }^{33}$ the Urdu 5D-Itch scale ${ }^{47}$ or other validated scales used to evaluate changes in $\mathrm{CP}$ after at least 2 weeks of treatment. ${ }^{48}$

\section{Secondary outcomes}

Secondary outcomes include the effective rate (Effective rate $(\%)=[$ (number of patients clinically cured + markedly effective+effective)/number of patients $] \times 100 \%$. (1) Clinically cured: The skin lesions have subsided, the pruritus has completely disappeared, and scores have decreased by 90\% or more after treatment; (2) Markedly effective: The skin lesions have significantly resolved, the pruritus has been significantly alleviated and scores have decreased by 60\%-89\%; (3) Effective: Skin lesions have resolved, the pruritus has been alleviated and scores have decreased by $20 \%-59 \%$; 4) Invalid: Skin lesions and pruritus were not significantly resolved, and scores have decreased by less than $20 \%,{ }^{490}$ Quality of Life Scale (eg, EuroQol fivedimension questionnaire (EQ-5D) third level, Dermatology Life Quality Index, etc), ${ }^{51}$ Pittsburgh Sleep Quality Index,${ }^{29}$ recurrence rate during the follow-up period and adverse events. The systematic review will be performed independently.

\section{Search methods for identification of studies \\ Electronic searches}

This systematic review will consist of an electronic and manual search for all RCTs for CP acupuncture treatment, published from the inception date of each predefined database up to 28 February 2020 without language and publication limitations. Databases include: PubMed, Embase, Springer, Web of Science, the Cochrane Library, the World Health Organization International Clinical Trials Registry Platform (ICTRP), TCM databases, the China National Knowledge Infrastructure, the China Biomedical Literature Database, the Chinese Scientific Journal Database and the Wan-Fang Database. The following search terms will be used: itch, itching, pruritus, CP, acupuncture, manual acupuncture, filiform 


\begin{tabular}{|c|c|}
\hline Number & Search terms \\
\hline 1 & Randomised controlled trial.pt \\
\hline 2 & Controlled clinical trial.pt \\
\hline 3 & Randomised.ti,ab \\
\hline 4 & Randomly.ti,ab \\
\hline 5 & Placebo.ti,ab \\
\hline 6 & Sham.ti,ab \\
\hline 7 & Trial.ti,ab \\
\hline 8 & Groups.ti,ab \\
\hline 9 & 1 or $2-8$ \\
\hline 10 & Acupuncture therapy. Mesh. \\
\hline 11 & Acupuncture.ti,ab \\
\hline 12 & Acupoints.ti,ab \\
\hline 13 & Body acupuncture.ti,ab \\
\hline 14 & Scalp acupuncture.ti,ab \\
\hline 15 & manual acupuncture.ti,ab \\
\hline 16 & Auricular acupuncture.ti,ab \\
\hline 17 & ear acupuncture.ti,ab \\
\hline 18 & Electroacupuncture.ti,ab \\
\hline 19 & Fire needling.ti,ab \\
\hline 20 & dermal needle.ti,ab \\
\hline 21 & plum blossom needle.ti,ab \\
\hline 22 & Pyonex.ti,ab \\
\hline 23 & Abdominal acupuncture.ti,ab \\
\hline 24 & Filiform steel needle.ti,ab \\
\hline 25 & 10 or $11-24$ \\
\hline 26 & Pruritus.Mesh \\
\hline 27 & Chronic pruritus.ti,ab \\
\hline 28 & itch.ti,ab \\
\hline 29 & itching.ti,ab \\
\hline 30 & Chronic itch.ti,ab \\
\hline 31 & 26 or $27-30$ \\
\hline 32 & 9 and 25 and 31 \\
\hline
\end{tabular}

steel needle, electroacupuncture, fire needling, auricular acupuncture, ear acupuncture, dermal needle, abdominal acupuncture, pyonex and plum blossom needle. The same search terms will be used in the Chinese database. A search strategy will be developed based on the Cochrane Handbook guidelines. ${ }^{52}$ The search strategy for the PubMed database is shown in table 1.

\section{Searching other resources}

The list of all identified publications, including relevant systematic reviews and meta-analyses, will be reviewed to further identify additional trials. Ongoing trials with unpublished data will be retrieved from the following clinical trial registries: the ICTRP (http://www.who. int/ictrp/en/), the National Institute of Health clinical

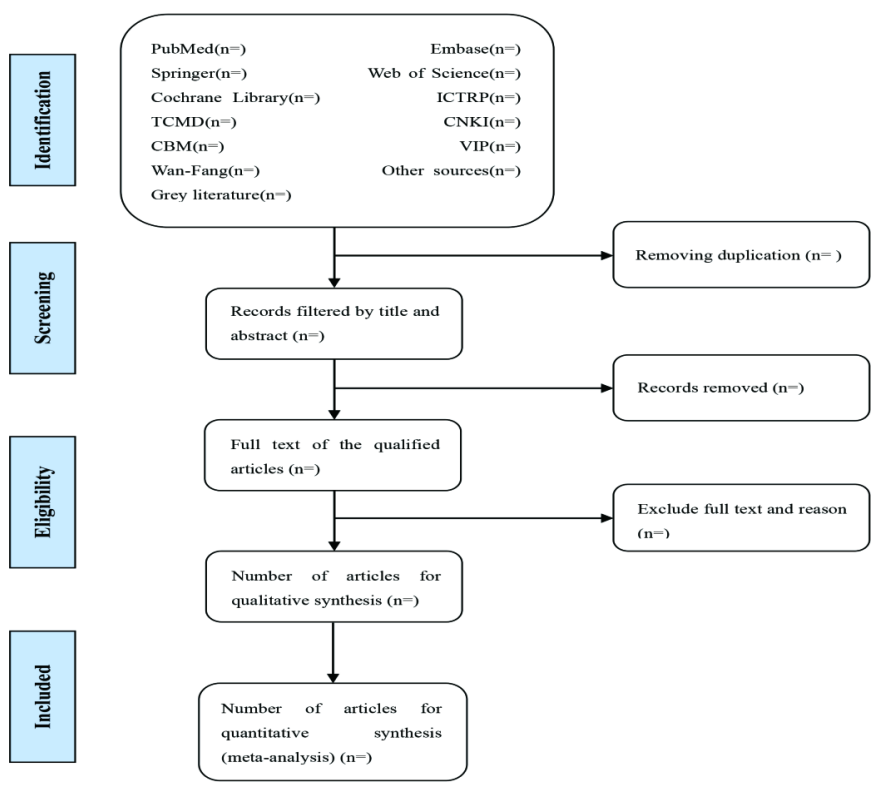

Figure 1 Flow diagram of studies identified. ICTRP, International Clinical Trials Registry Platform. CTRP, International Clinical Trials Registry Platform; TCMD, Traditional Chinese Medicine Database; CNKI, China National Knowledge Infrastructure; CBM, China Biomedical Literature Database; VIP, Chinese Scientific Journal Database.

registry ClinicalTrials.gov (https://www.clinicaltrials. gov/), the Chinese clinical registry (http://www.chictr. org/en/) and the Australian New Zealand Clinical Trials Registry (http:/ /www.anzctr.org.au/). For ongoing RCTs, the author of the trial will be contacted for the most up-todate clinical data. The search will also include meeting minutes related to this topic, and a manual search for grey literature including unpublished conference articles.

\section{DATA COLLECTION AND ANALYSIS Selection of studies}

All reviewers are trained to ensure a good understanding of the background and purpose of the review. After electronic retrieval, all records will be uploaded to the database created using the EndNote software (V.X8). Records selected manually or from other sources will be moved to the same database. The two review authors (LZ and YD) will independently screen the titles, abstracts and keywords of all retrieved trials and determine which trials meet the afore-mentioned inclusion criteria. We will obtain the full text of all relevant trials for further review and evaluation. Excluded trials will be recorded and explained. Any dispute will be resolved by the two authors (LZ and $\mathrm{YD})$ and a third author (YLi), and an arbitration procedure will be used if necessary. If required, we will contact the trial author for clarification. The primary selection process is shown in a PRISMA flow chart (figure 1).

\section{Data extraction and management}

Two investigators will extract data independently from the selected report or study, and complete a data extraction form. Data regarding general information, reference 
identification, publication year, first author, participants, sample size, randomisation, allocation concealment and blinding methods, intervention type, control intervention type, outcomes, follow-up duration, adverse events, conflicts of interest, ethical perceptions and a list of the Standards for Reporting Interventions in Controlled Trials of Acupuncture will be obtained. When the reported data are insufficient, we will contact the author for more information. Any disagreement will be resolved by a discussion between the two authors and any further disagreement will be arbitrated by the third author (YLi).

\section{Risk of bias assessment in included studies}

The authors (JY and XX) will use the Cochrane Collaboration's bias risk assessment tool to assess the risk of bias for all included studies. We will assess the risk of bias in sequence generation; allocation sequence concealment; the blinding of participants and staff, and outcome assessors; incomplete outcome reporting; selective reporting of results; and other sources of deviation. This review uses $\mathrm{L}, \mathrm{U}$ and $\mathrm{H}$ as the key to these assessments, where $\mathrm{L}$ (low) indicates a lower risk of bias, $\mathrm{U}$ (unclear) indicates an uncertain risk of bias and $\mathrm{H}$ (high) indicates a higher risk of bias. If inconsistent results occur, the final decision will be made by the third author (YLi). The information contained in the study on the risk of bias assessment will be summarised in tabular form, and the results and impacts will be critically discussed. If the information is not clear, we will attempt to contact the author. For duplicate articles, only the original will be used.

\section{Measures of treatment effects}

Data regarding effectiveness will be synthesised and statistically analysed in RevMan V.5.3. For continuous data, the mean difference (MD) or standard MD will be used to measure the therapeutic effect with $95 \%$ CIs. For dichotomous data, risk ratios with $95 \%$ CIs will be calculated.

\section{Unit of analysis issues}

We will include a meta-analysis of data from parallelgroup design studies. In these trials, participants are randomly assigned to two intervention groups individually, and individual measurements for each outcome of each participant are collected and analysed.

\section{Dealing with missing data}

We will attempt to contact the first author or corresponding author of the study to request for missing or insufficient data. If possible, an intent-to-treat analysis (including data from all participants) will be performed, and a sensitivity analysis will be used to determine if the results are inconsistent.

\section{Heterogeneity assessment}

According to the Cochrane Handbook for Systematic Reviews of Interventions, heterogeneity can be assessed by a visual check of the forest plot, a heterogeneity $\chi^{2}$ test and Higgins' $\mathrm{I}^{2}$ statistic. If the $\mathrm{p}$ value is $>0.10$ and the $\mathrm{I}^{2}$ value is $<50 \%$, a fixed-effects model will be used to pool the data. Otherwise, a random-effects model will be used. When heterogeneity is identified, a meta-analysis with the random-effect model will be used to estimate the overall treatment effect. Moreover, a subgroup analysis or metaregression will be conducted to explore the causes of heterogeneity among the study results.

\section{Reporting bias assessment}

The funnel plot is used to detect reported bias and the effects of small-scale studies. If there are more than 10 studies, Begg's and Egger's tests will be performed using the Stata V.14.0 software to assess the asymmetry of the funnel plot. A value of $\mathrm{p}<0.05$ will indicate a significant publication bias. ${ }^{5354}$ All eligible trials, regardless of the quality of their methods, will be included.

\section{DATA SYNTHESIS}

When the meta-analysis is performed, RevMan V.5.3 (Copenhagen: The Nordic Cochrane Centre, The Cochrane Collaboration, 2014) will be used for data synthesis. The use of a fixed-effects or random-effects model will be determined based on the heterogeneity level. The fixed-effects model will be used if little or no statistical heterogeneity is found among the trials. On the other hand, the random-effects model will be used for data synthesis if significant heterogeneity (the $\mathrm{I}^{2}$ value is not $<50 \%)$ is observed. If there is considerable heterogeneity in the trials, a meta-analysis will not be performed. In this case, we will provide a descriptive qualitative summary. If necessary, each subgroup will be carefully considered for subgroup analyses.

\section{SUBGROUP ANALYSIS}

Subgroup analyses will be performed based on the heterogeneity of the acupuncture type (including manual acupuncture, dermal needle, plum blossom needle, ear acupuncture, electroacupuncture or fire needle) and clinical differences.

\section{SENSITIVITY ANALYSIS}

To test the robustness of the review conclusions, a sensitivity analysis will be performed for the primary outcome according to the following criteria: sample size, heterogeneity quality and statistical model (random-effects or fixed-effects model). The results will be compared and discussed.

\section{Grading the quality of evidence}

The Grades of Recommendation, Assessment, Development and Evaluation approach will be used to describe the quality of the evidence for the results obtained. The following factors will be assessed: risk of bias, heterogeneity, indirectness, imprecision and publication bias. The assessment will be divided into four levels: high, moderate, low and very low. 


\section{Discussion}

Many studies have shown that acupuncture is effective in relieving CP, and has no significant side effects. ${ }^{28} 304055$ However, no systematic reviews on this topic have been published. Therefore, we believe that it is necessary to provide a comprehensive review of the relevant studies published to date. The evaluation will consist of four parts: identification, inclusion studies, data extraction and data synthesis. The limitation of this protocol is that there may be heterogeneity among the studies due to the use of different evaluation criteria and acupuncture methods. This systematic review is intended to provide clinicians with more supportive evidence in the decisionmaking process for $\mathrm{CP}$ treatment.

\section{Ethics and dissemination}

Since we are not directly targeting individuals or extracting data without privacy, formal ethical approval is not necessary. The results of the study will be disseminated through peer-reviewed publications or conference reports.

\section{Patient and public involvement}

Patient priorities, experiences and preferences were not involved in the development of the research question, outcome measures, study design or conduct of this review. The results will not be disseminated to study participants.

Acknowledgements We would like to thank Youping Hu for his assistance in improving this manuscript.

Contributors YLi is the guarantor. LZ, YLi and JY contributed to the concept of the study. QZ, SZ and SY developed the protocol methodology. HZ and PH were responsible for the planning of the statistical analysis. WC, YLiu and YS monitored the implementation of the protocol. The original manuscript was jointly completed by $L Z$ and YD, and modified by YLi. Authors LZ and YD will independently screen potential studies, and extract data from the included studies. JY and XX will assess risk of bias and perform data synthesis. YLi will arbitrate any conflicts between reviewers, and perform quality checks at all stages of the review. All authors read, provided feedback and approved the final manuscript.

Funding This study was supported by the National Key Research and Development Program of the China-Key Project 'Research on Modernization of Traditional Chinese Medicine'-'International Cooperation Research on Evaluation of Acupuncture Advantage Diseases' (grant number 2017YFC1703605) (awarded to YLi).

Competing interests None declared.

Patient consent for publication Not required.

Provenance and peer review Not commissioned; externally peer reviewed.

Open access This is an open access article distributed in accordance with the Creative Commons Attribution Non Commercial (CC BY-NC 4.0) license, which permits others to distribute, remix, adapt, build upon this work non-commercially, and license their derivative works on different terms, provided the original work is properly cited, appropriate credit is given, any changes made indicated, and the use is non-commercial. See: http://creativecommons.org/licenses/by-nc/4.0/.

\section{ORCID iDs}

Leixiao Zhang http://orcid.org/0000-0002-8117-6213

Hui Zheng http://orcid.org/0000-0002-0494-1217

\section{REFERENCES}

1 Ständer S, Weisshaar E, Mettang T, et al. Clinical classification of itch: a position paper of the International forum for the study of itch. Acta Derm Venereol 2007;87:291-4.
2 Matterne U, Apfelbacher CJ, Loerbroks A, et al. Prevalence, correlates and characteristics of chronic pruritus: a population-based cross-sectional study. Acta Derm Venereol 2011;91:674-9.

3 Weisshaar E, Szepietowski JC, Darsow U, et al. European guideline on chronic pruritus. Acta Derm Venereol 2012;92:563-81.

4 Weisshaar E, Dalgard F. Epidemiology of itch: adding to the burden of skin morbidity. Acta Derm Venereol 2009;89:339-50.

5 Millington GWM, Collins A, Lovell CR, et al. British association of dermatologists' guidelines for the investigation and management of generalized pruritus in adults without an underlying dermatosis, 2018. Br J Dermatol 2018;178:34-60.

6 Ständer S, Schäfer I, Phan NQ, et al. Prevalence of chronic pruritus in Germany: results of a cross-sectional study in a sample working population of 11,730. Dermatology 2010;221:229-35.

7 Berger TG, Shive M, Harper GM. Pruritus in the older patient: a clinical review. JAMA 2013;310:2443-50.

8 Beauregard S, Gilchrest BA. A survey of skin problems and skin care regimens in the elderly. Arch Dermatol 1987;123:1638-43.

9 Yalçin B, Tamer E, Toy GG, et al. The prevalence of skin diseases in the elderly: analysis of 4099 geriatric patients. Int J Dermatol 2006;45:672-6.

10 Thaipisuttikul Y. Pruritic skin diseases in the elderly. J Dermatol 1998;25:153-7.

11 Shive M, Linos E, Berger T, et al. Itch as a patient-reported symptom in ambulatory care visits in the United States. J Am Acad Dermatol 2013;69:550-6

12 Hartmann EM, Handwerker HO, Forster C. Gender differences in itch and pain-related sensations provoked by histamine, cowhage and capsaicin. Acta Derm Venereol 2015;95:25-30.

13 Damiani G, Cazzaniga S, Conic RR, et al. Pruritus characteristics in a large Italian cohort of psoriatic patients. J Eur Acad Dermatol Venereol 2019;33:1316-24.

14 Weisshaar E, Diepgen TL, Luger TA, et al. Pruritus in pregnancy and childhood--do we really consider all relevant differential diagnoses? Eur J Dermatol 2005;15:320-31.

15 Girling JC. Obstetric cholestasis. guideline no 43. London: Royal College of Obstetricians and Gynaecologists (RCOG), 2006.

16 Halvorsen JA, Dalgard F, Thoresen M, et al. Itch and pain in adolescents are associated with suicidal ideation: a populationbased cross-sectional study. Acta Derm Venereol 2012;92:543-6.

17 Halvorsen JA, Dalgard F, Thoresen M, et al. Itch and mental distress: a cross-sectional study among late adolescents. Acta Derm Venereol 2009;89:39-44.

18 Yosipovitch G, Goon ATJ, Wee J, et al. Itch characteristics in Chinese patients with atopic dermatitis using a new questionnaire for the assessment of pruritus. Int J Dermatol 2002;41:212-6.

19 Szepietowski JC, Reich A, Wiśnicka B. Itching in patients suffering from psoriasis. Acta Dermatovenerol Croat 2002;10:221-6.

20 Szepietowski JC, Reich A, Wisnicka B. Pruritus and psoriasis. Br J Dermatol 2004;151:1284.

21 Kaplan AP. Drug-Induced skin disease. J Allergy Clin Immunol 1984;74:573-9.

22 Metze D, Reimann S, Szepfalusi Z, et al. Persistent pruritus after hydroxyethyl starch infusion therapy: a result of long-term storage in cutaneous nerves. Br J Dermatol 1997;136:553-9.

23 Pfab F, Valet M, Sprenger T, et al. Temperature modulated histamineitch in lesional and nonlesional skin in atopic eczema - a combined psychophysical and neuroimaging study. Allergy 2010;65:84-94.

24 Zuberbier T, Aberer W, Asero R, et al. The EAACl/GA(2) LEN/EDF/ WAO Guideline for the definition, classification, diagnosis, and management of urticaria: the 2013 revision and update. Allergy 2014;69:868-87.

25 Saruwatari J, Matsunaga M, Ikeda K, et al. Impact of CYP2D6*10 on $\mathrm{H} 1$-antihistamine-induced hypersomnia. Eur J Clin Pharmacol 2006;62:995-1001.

26 Simons FER, Simons KJ. Histamine and H1-antihistamines: celebrating a century of progress. J Allergy Clin Immunol 2011;128:1139-50

27 Lim HW, Collins SAB, Resneck JS, et al. The burden of skin disease in the United States. J Am Acad Dermatol 2017;76:958-72.

28 Manway M, Blackburn S, Barr J, et al. Effects of acupuncture on chronic idiopathic pruritus: an uncontrolled pilot study evaluating inflammatory changes with treatment. J Complement Integr Med 2018;16:1-5.

29 Xiong W, He F-F, You R-Y, et al. Acupuncture application in chronic kidney disease and its potential mechanisms. Am J Chin Med 2018;46:1169-85.

30 Mazda Y, Kikuchi T, Yoshimatsu A, et al. Acupuncture for reducing pruritus induced by intrathecal morphine at elective cesarean delivery: a placebo-controlled, randomized, double-blind trial. Int J Obstet Anesth 2018;36:66-76. 
31 Weisshaar E, Szepietowski JC, Dalgard FJ, et al. European S2k guideline on chronic pruritus. Acta Derm Venereol 2019;99:469-506.

32 Yu C, Zhang P, Lv Z-T, et al. Efficacy of acupuncture in itch: a systematic review and meta-analysis of clinical randomized controlled trials. Evid Based Complement Alternat Med 2015;2015:1-5.

33 Badiee Aval S, Ravanshad Y, Azarfar A, et al. A systematic review and meta-analysis of using acupuncture and Acupressure for uremic pruritus. Iran J Kidney Dis 2018;12:78-83.

34 Wu JN. A short history of acupuncture. J Altern Complement Med 1996;2:19-21.

35 Cheuk DKL, Yeung W-F, Chung KF, et al. Acupuncture for insomnia Cochrane Database Syst Rev 2012:CD005472.

36 Maciocia G. Foundations of Chinese medicine: a comprehensive tex for Acupuncturists and Herbalists. Edinburgh: Churchill Livingstone, 1989.

37 Napadow V, Li A, Loggia ML, et al. The brain circuitry mediating antipruritic effects of acupuncture. Cereb Cortex 2014;24:873-82.

38 Melzack R, Wall PD. Pain mechanisms: a new theory. Science 1965;150:971-8.

39 Min S, Kim K-W, Jung W-M, et al. Acupuncture for histamineinduced itch: association with increased parasympathetic tone and connectivity of Putamen-Midcingulate cortex. Front Neurosci 2019;13:215.

40 Kang S, Kim Y-K, Yeom M, et al. Acupuncture improves symptoms in patients with mild-to-moderate atopic dermatitis: a randomized, sham-controlled preliminary trial. Complement Ther Med 2018;41:90-8.

41 Shamseer L, Moher D, Clarke M, et al. Preferred reporting items for systematic review and meta-analysis protocols (PRISMA-P) 2015 elaboration and explanation. BMJ 2015;350:g7647.

42 Liberati A, Altman DG, Tetzlaff J, et al. The PRISMA statement for reporting systematic reviews and meta-analyses of studies that evaluate healthcare interventions: explanation and elaboration. BMJ 2009;339:b2700.

43 Long BJ, Koyfman A, Gottlieb M. Evaluation and management of angioedema in the emergency department. West $J$ Emerg Med 2019;20:587-600.
$44 \mathrm{He} \mathrm{G}$, Ruan J, Lv X, et al. [Analysis on the atypical clinical accidents of acupuncture based on the individual factors in patients]. Zhongguo Zhen Jiu 2018;38:1245-8.

45 Jiang $Y$, Yin L, Wang Y, et al. Assessments of different kinds of sham acupuncture applied in randomized controlled trials. J Acupunct Tuina Sci 2011;9:199-203.

46 Fei YT, Guo ZN, Chai QY, et al. Methodology key points and quality control in randomized controlled trials of needling. J Beijing Univ Tradit Chin Med 2013:27-30.

47 Rehman IU, Bin-Chia Wu D, Ahmed R, et al. A randomized controlled trial for effectiveness of zolpidem versus acupressure on sleep in hemodialysis patients having chronic kidney disease-associated pruritus. Medicine 2018;97:e10764.

48 Yang M, Du T, Sun M, et al. Acupuncture for stable angina pectoris: a systematic review protocol. BMJ Open 2018;8:e019798.

49 Nie L, Cheng J, Wen Y, et al. The effectiveness of acupuncture combined with Tuina therapy in patients with migraine. Complement Med Res 2019;26:182-94.

50 Standards for TCM industry of the People's Republic of China. Criteria of diagnosis and therapeutic effect of diseases and syndromes in TCM (ZY/T001.1-94). Nanjing University Press, 1996.

51 Ständer S, Augustin M, Reich A, et al. Pruritus assessment in clinical trials: consensus recommendations from the International forum for the study of itch (IFSI) special interest group scoring itch in clinical trials. Acta Derm Venereol 2013;93:509-14

52 Higgins JPT, Green S. Cochrane Handbook for systematic reviews of interventions version 5.1.0. The Cochrane collaboration, 2011. Available: http://www.cochrane-handbook.org

53 Deeks JJ, Higgins JPT, Altman DG. Analysing data and undertaking meta-analysis. In: Higgins JPT, Green S, eds. Cochrane handbook for systematic reviews of interventions version 5.1.0 [updated March 2011]. The Cochrane Collaboration,, 2011. Available: http://www. cochrane-handbook.org [Accessed Jun 2014].

54 Egger M, Davey Smith G, Schneider M, et al. Bias in meta-analysis detected by a simple, graphical test. BMJ 1997;315:629-34.

55 Pfab F, Huss-Marp J, Gatti A, et al. Influence of acupuncture on type I hypersensitivity itch and the wheal and flare response in adults with atopic eczema - a blinded, randomized, placebo-controlled, crossover trial. Allergy 2010;65:903-10. 